\title{
Modeling Context Information for Capture and Access Applications
}

\author{
Laercio Baldochi, Maria Graça Pimentel \\ \{baldochi,mgp\}@icmc.usp.br \\ Depto Computação \\ ICMC-Universidade de São Paulo \\ 13560-970 São Carlos, SP Brazil
}

\author{
Ethan V. Munson \\ munson@uwm.edu \\ Dept. of EECS \\ University of Wisconsin-Milwaukee \\ Milwaukee, WI 53211 USA
}

\begin{abstract}
Ubiquitous computing applications rely heavily on context information to work properly. Capture and access applications, a special type of ubiquitous computing applications, process context information during the live capture of experiences and, afterwards, use this information to retrieve, present and extend the captured content. The Contextractor is an XSLT-based transformation system that gathers context information from extended UML models. The XSLT transformations produce XML Schemas that model the context information produced by the capture and access application and define a contextual query language that allows the submission of contextual queries over the captured content. We also provide an automated system that allows the presentation of query results on the Web in different formats such as HTML, SMIL and HTML+SMIL.
\end{abstract}

\section{Keywords}

UML models, XSLT transformations, capture and access applications

\section{INTRODUCTION}

In the ubiquitous computing (ubicomp) paradigm, users interact with everyday devices that transparently embed applications. The main idea, as proposed by Weiser [18], is to support users in everyday tasks, which are usually carried out away from the desktop platform.

There are three recurring themes in ubiquitous computering research: natural interfaces, context awareness, and capture and access applications [1]. Natural interfaces are those that support natural human interaction. Context aware applications can identify users and objects and then adapt the environment to suit user needs. Capture and access (C\&A) applications capture live experiences, such as lectures and meetings, and then organize the captured material as docu- ments for later review.

These three themes are not isolated. For instance, a C\&A application usually presents natural interfaces and processes context information. However, while there have been solid research results for the intersection between natural interfaces and C\&A applications, the use of contextual information in C\&A applications is still at an embryonic stage.

Ongoing research on context awareness is mainly focused on supporting smart environments, where sensors and actuators are used to monitor the situational state of people and objects. Smart environment applications typically process a large amount of context data, which must be reliably and efficiently stored, queried and delivered in order to provide the expected pervasive facilities. As an example of such environment, the ParcTab System [17] was a pioneer in the exploitation of location awareness, allowing access to files linked to a particular location. As users moved between different locations, a file browser would change to display relevant data. For C\&A applications, however, these needs are quite different.

In a C\&A application, the capture and access phases are rather distinct. The capture phase is centered around information acquisition during live experiences. It closely resembles a typical ubicomp application because the classroom or meeting room is a smart environment and correct behavior of the application depends primarily on identifying the location of an activity and the people and objects in that location. In contrast, the access phase more closely resembles a desktop application, with only one person accessing the information using a conventional desktop or laptop computer.

During access, the system must provide efficient ways to retrieve captured data based on context information acquired during the capture phase. Knowing that user C45F32 was present in a given captured experience is not enough. It is necessary to annotate people, places and objects in order to provide efficient retrieval capabilities during access. If the captured activity is a class, it is important to know whether user C45F32 is an instructor or a student. If she is an instructor, it is desirable to know if she is the profesor responsible for the course, or just an invited speaker. These sorts of annotations are domain-specific, so C\&A systems must have information about the application domain 
in order to model useful contextual information. Prior ubicomp research has not addressed this need and we observe a corresponding lack of tools.

To correct this situation, we have built the Contextractor, a system that automates many aspects of the construction of context-aware C\&A applications. To use the Contextractor, the developer of a C\&A application creates a UML class diagram that models the domain of the captured activity, such as a classroom, meeting, or party. This class diagram is saved using the XML-based XMI format. Then, building upon previous work by Andrade et al. [2, 3], Contextractor uses XSLT transformations to translate this XMI document into a set of XML Schemas. These schemas define a domainspecific storage representation for the captured content and a corresponding query language to be used when accessing this content.

Our approach to extracting context information from application domain models is novel in the ubiquitous computing field because of its pervasive use of document technology, including XMI, XSLT, XML Schema, and XQuery. The Contextractor uses these technologies to model, store, and retrieve captured content using concepts that are specific to the capture environment. This makes later access by end users more natural because they can use concepts appropriate to the captured activity, rather than generic C\&A concepts.

This paper is organized as follows. Section 2 presents an onverview of our approach, discussing the motivation for the Contextractor and how it fits in a larger project called InCASERVE. Section 3 presents our approach to map contextual dimensions to UML attributes. Section 4 presents the XSLT transformations that produces XML schemas from extended UML models. Section 5 discusses how these schemas are used by the InCA-SERVE infrastructure to produce XML documents that represents C\&A applications. Section 6 presents the CQL processor, a system that exploits the XML schemas produced by the Contextractor to provide contextual query facilities for accessing and presenting captured content. Section 7 discuss related work and Section 8 presents our final remarks.

\section{CONTEXTRACTOR OVERVIEW}

In order to model a captured session, Abowd and Mynatt [1] defined 5 context dimensions: who are the users involved in the experience, what is being captured (a lecture, a meeting, etc.), where the experience takes place, when it happens and why. Regardless of the captured experience, it is often possible to associate these dimensions with the captured data: each experience has a begin and an end, occurs in a certain place, in most of the cases, involve a number of different people, and can be annotated by a name associated with the performed activities.

The literature reports the use of context dimensions to support different C\&A applications (e.g. [16]). However, there is a lack of a model or methodology to map application data to context dimensions. We believe that a formal procedure to aid the specification of context information is important to support data management in $\mathrm{C} \& \mathrm{~A}$ applications, especially when processing the captured content. Helal has called for similar work to support the programming of pervasive spaces [12].

Given this requirement, we developed the Contextractor, a system that gathers context information from UML models. This approach allows the modeling of context information using a standard modeling tool, with a few extensions, and imposes only a minimal learning curve on the application developer. The Contextractor extends ArgoUML, an open source UML modeling tool, so that an application developer can associate attributes of the application model with the who, where and when context dimensions. The what context dimension is automatically derived from the application model. Exported as an XMI document, this extended class diagram is used as input to a set of XSLT transformations that produce three XML schemas. The first schema, called context-info, is used to model the context information manipulated by the application. The other two schemas model a contextual query language that allows the retrieval of captured data based on the four context dimensions.

The context-info schema is used as a building block of InCA$S E R V E$, an infrastructure that supports the development of C\&A applications $[4,5,6,9]$. In $C A-S E R V E$ provides high level software components that capture user interaction with input devices such as electronic whiteboards, tablet PCs, microphones and video cameras. A special software component is also capable of capturing Web browsing. The information produced by these components is modeled using XML schemas, which serve as input for a data binding mechanism that produces classes responsible for holding the data generated by each component.

The programming model offered by the InCA-SERVE infrastructure allows the construction of a C\&A application by simply instantiating appropriate software components. To provide facilities for storing the captured content, the Contextractor system includes the Transducer, a piece of software that receives the data captured by a C\&A application and produces an XML document representing the captured content. The Transducer implements the same set of objects present in the C\&A application. During the capture phase, the capture components send captured objects to the Transducer, which assembles them in a data structure that, by the end of the capture session, produces an XML document using the facilities provided by the data binding mechanism.

The context-info schema produced by the Contextractor is used by the Transducer to include context information in the session document. As with the other schemas, contextinfo is compiled by the data binding mechanism - resulting classes are used by the C\&A application to pass the context information to the Transducer. In this way, the session document produced by the Transducer encapsulates all the information produced by the C\&A application.

The InCA-SERVE infrastructure maintains a repository where the session documents are stored. The other two schemas produced by the Contextractor formalize a query language for the C\&A application, which is used to submit query expressions to the document repository. The response to each query is a list containing references to the documents that 
obey the restrictions specified in the query.

\section{MAPPING CONTEXT INFORMATION}

Observing the several applications we have built during the last three years we noticed that the context information relevant to a given application is usually kept on a legacy system. For example, C\&A applications targeted to the classroom typically use an academic information system that stores data about instructors, students, courses and classrooms. A C\&A application for meetings might retrieve data about users, projects and rooms from a corporate database. So, instead of modeling a C\&A application from scratch, context information may be obtained from existing legacy models. However, our approach also works for applications completely built from scratch.

Figure 1 shows the UML model for a classroom C\&A application. This model uses information about courses, teachers and students stored in an academic information system. The composition relationship represents the junction between the information stored in the legacy system and the data produced by a C\&A application.

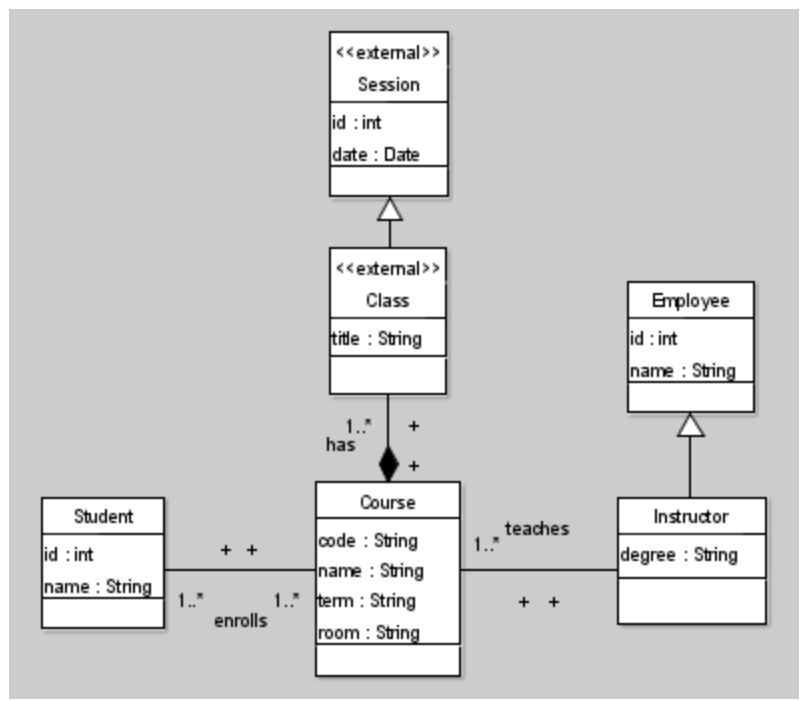

Figure 1: Class diagram for a $C \& A$ application targeted to the classroom domain.

The model shown on Figure 1 is only illustrative, since a session is composed of many other classes associated with C\&A components. We use this simplified model so that our discussion can focus on the processing of the context information.

To map the context dimensions from the entities of the UML model, we used stereotypes and tagged values, which are part of the UML extension mechanism. UML provides an extension mechanism to allow the modeling of applications for specific domains. For the capture and access domain, we use the extension mechanisms to specify whether a class is internal or external, i.e., if it belongs to the legacy system or not, and also to associate attributes with the context dimensions who, where and when.

To annotate classes, we used stereotypes because they make it easier to visualize the external classes in the class diagram, as it is shown in Figure 1. This procedure is important to define the dimension what.

The association between attributes and context dimensions is done using tagged-values. The creation of a tagged-value involves the selection of an attribute of a given class, the specification of a tag name and its associated value. Using this procedure, one may select the room attribute of the class Course, create the tagged value context-dimension and set its value to where. Despite of being effective in the modeling of the context information, this procedure does require the developer to understand the UML extension mechanisms and how these mechanisms are implemented in the UML modeling tools.

We have extended the open source modeling tool ArgoUML to provide new GUI items for creating the stereotypes and tagged-values needed for the modeling of $\mathrm{C} \& \mathrm{~A}$ applications. To allow the user to set the stereotype external, we included the External check box in the class pop-up menu. Another extension was made in the attribute properties, where we included a set of radio buttons that allows the association of a context-dimension to a given attribute by simply selecting the desired button (Figure 2).

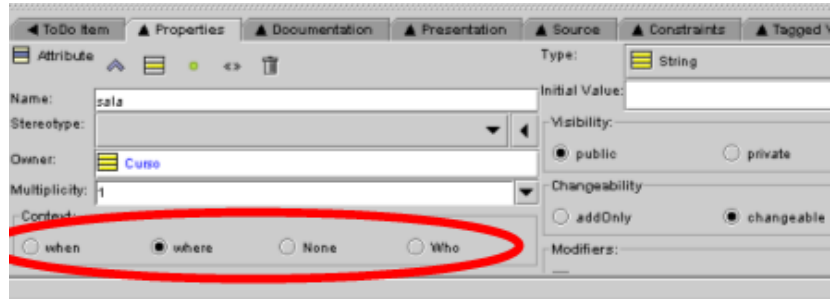

Figure 2: Extension in ArgoUML.

The selection of the radio button where, as shown in the figure, automatically creates a tagged-value for the current selected attribute with the name context-dimension and the value where. A similar procedure happens when the user selects the option External for a given class.

At the end of the modeling activity, the user must export the resulting model as an XMI document.

\section{XSLT TRANSFORMATIONS}

The XMI document produced by the end of the modeling phase describes all the details present in the graphical model, including the extensions added by means of the stereotypes and tagged-values. Using this document as input for a pipeline of XSLT transformations, the Contextractor produces three XML schemas that model the context data used by the $\mathrm{C} \& \mathrm{~A}$ application and defines a contextual query language used to retrieve captured content.

The overview of the transformation process specified by the Contextractor is shown in Figure 3. Larger documents represent XML content, smaller ones are XSLT stylesheets.

The transformation process starts with the generation of the application's conceptual model (CM), obtained by filtering the XMI document. This filtering reduces the verbosity of 


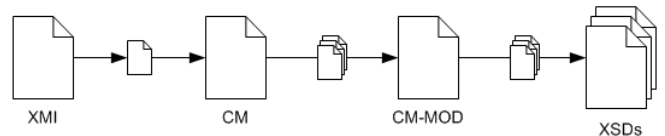

Figure 3: Pipeline of XSLT transformations.

the XMI document, exposing the application's conceptual model.

The next transformation prepares the conceptual model to be mapped to an underlying storage model. In this phase, the relationships between the conceptual classes are processed, producing the modified conceptual document (CMMOD in Fig. 3).

The generation of the modified conceptual model is achieved in three steps, using three distinct stylesheets. The first stylesheet processes the generalization relationships, generating $i d$ attributes in the generalized classes when needed. The second stylesheet adjusts the context dimensions of the attributes involved in generalization relationships. The third stylesheet processes all other relationships, producing new attributes to reflect the multiplicity of these relationships.

The modified conceptual model is processed in the final transformation to generate XML schemas (XSDs) used for modeling both (a) the application's context information and (b) a contextual query language used to retrieve the content produced by the modeled application.

\subsection{Generating the conceptual model}

The first transformation takes the XMI document as input and produces the application's conceptual model. We present some fragments of the CM document below.

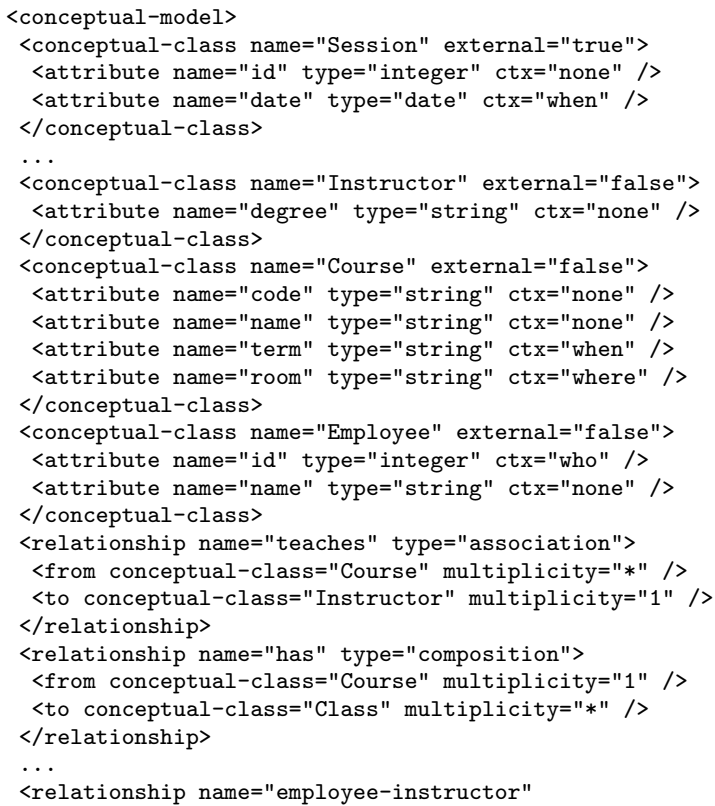

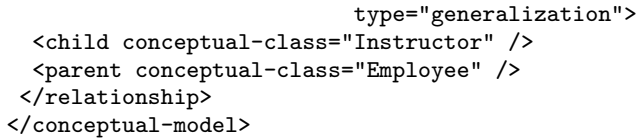

This code fragment shows that classes and attributes in the UML model were mapped to the XML elements conceptualclass and attribute, respectively. The extensions to the native model appear as attributes in the CM document: the stereotype external was mapped to an attribute of the element conceptual-class, and the tagged-value context-dimension became the attribute ctx of the element attribute.

The next step towards generating the XML schemas involves the analysis of the relationships present in the conceptual model.

\subsection{The modified conceptual model}

Unless object-oriented databases are used, it is not possible to map the generated conceptual model directly to an underlying storage model. However, with a few modifications it is possible to do such a mapping to the relational model, or even to a storage model based on XML documents. The required modifications involve analysis and modification of the relationships between the conceptual classes.

The modified conceptual model is produced to couple with existing implementations of the internal part of the C\&A application model. When there is no legacy system involved, the modified conceptual model is used to generate the storage model for the C\&A application. This case is discussed in the work of Andrade et al. [2].

Figure 4 shows the three-step transformation pipeline that generates the modified conceptual model (CM-MOD). The steps of the pipeline are controlled by three XSLT stylesheets, represented in Figure 4 by the small documents. Naturally, the intermediary steps of the pipeline also produce some temporary XML documents.

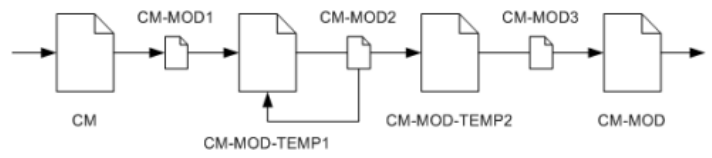

Figure 4: Modified conceptual model generation.

The first transformation handles the generalization relationships, making child classes inherit identifier attributes from parent classes. This procedure allows mapping both classes involved in a generalization to distinct tables in the relational model. This transformation only considers relationships that involve internal classes. For external classes, the generalization relationship is made explicit in XML documents by the nesting of elements.

The resulting document, CM-MOD-TEMP1, presents changes in just one element, as it is shown below.

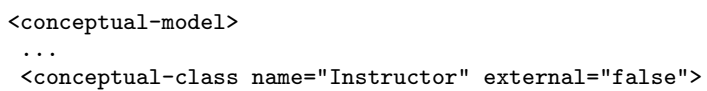




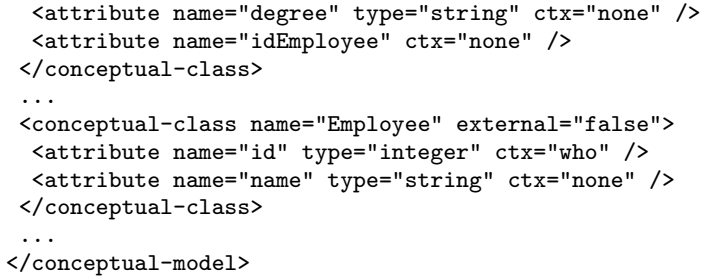

For our classroom example, the conceptual-class Instructor inherits the attribute idEmployee from the parent class Employee. One thing to notice in the above XML fragment is that the inherited attribute does not come with the contextdimension set to who, as it happens in the parent class. However, observing the application model shown on Figure 1, it becomes clear that the class Instructor must inherit the dimension who.

This fact emphasizes an important issue regarding the representation of context information in UML class diagrams: some context dimensions may be associated with classes, while others may only be associated with class attributes. For example, the class Student may be associated with the dimension who, while the dimension when may only be associated with attributes: term, in class Course and date in class Session.

As a way to make the procedure of associating entities from the UML class diagram to context dimensions uniform, we decided to associate the context with the identifier attribute of a class, every time that the context dimension is associated with a class as a whole.

This decision, however, presents a drawback: child classes of generalization relationships do not present identifier attributes. For these cases, when there is a context dimension associated with the child class, it is necessary to set the context dimension in the parent class and, afterwards, move the context dimension value to the child class during the transformation process.

This is exactly what happens with the class Instructor in our example. However, it is impossible to determine if a context dimension set in the parent class should stay there or be moved to a child class. To solve this issue, we provide a stylesheet (CM-MOD2) that moves the context annotation from the parent to the child class. This is the only stylesheet that requires a parameter, which must be the name of the parent class that owns the annotation that should be moved. When this transformation is done without parameters, the resulting document is identical to the input one.

One thing to observe about this transformation is that it treats one generalization relationship in each processing. So, this transformation should be repeated until all relationships are processed. For this reason, Figure 4 presents the cycle in the second step of the pipeline.

Finally, it is necessary to address the other relationships, as their multiplicities affect the mapping of classes to storage entities, such as tables in the relational model. The processing of relationships with multiplicity many to one causes the inclusion of an attribute in the class with the many multiplicity. For relationships in which both classes present multiplicity many to many, the processing results in the generation of a new relation containing the identifier attributes of both classes.

The following XML fragment shows the conceptual class Course and the relationship enrolls, which present modifications after the third transformation. In the conceptual class, the XSLT processing has produced a new attribute, $i d$ Employee, which has been included because of the many to one relationship between the classes Course and Instructor. In the case of the relationship enrolls, the inclusion of the attributes idCourse and idStudent happens because of the multiplicity many to many of this relationship.

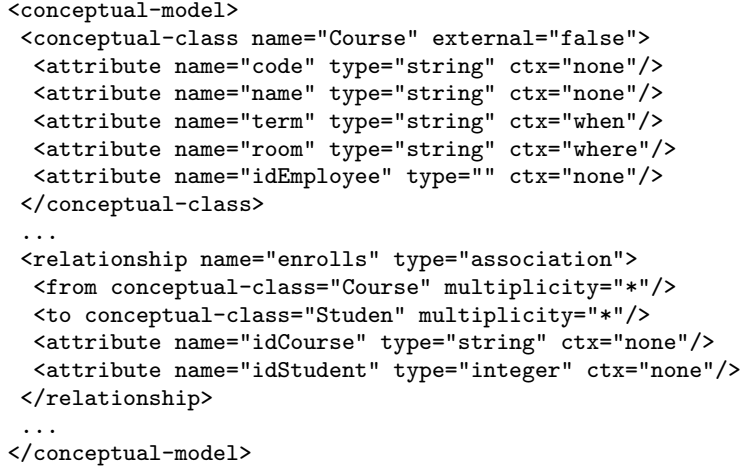

Is is worth noticing that the attributes $i d E m p l o y e e$ and $i d-$ Student, included, respectively, in the conceptual class Course and in the relationship enrolls do not preserve the value of their context dimension. This happens because a dimension that refers to the same information cannot be duplicated in different classes of the conceptual model.

The document produced by the end of this phase is the modified context model - CM-MOD in Figure 4. This document is next used to produce the XSDs that model the C\&A application's context information.

\subsection{Generating the XML schemas}

The final step of the Contextractor process takes as input the modified context model and produces the three XML schemas shown on Figure 5. The CONTEXT-INFO schema is used to model the application's context information. The other two schemas, CONTEXT-QUERY and FLWOR-QUERY are used to validate query expressions based on the modeled context dimensions.

The information in the UML extensions that were described in Section 3 guides the transformations that produce the XSD documents. Stereotypes annotate the conceptual classes in external and internal - this annotation is used to determine the dimension what.

As can be seen in Figure 2, it is possible to associate the dimensions who, where and when with a given attribute. A button for the what dimension is not included in the GUI because the what dimension can be extracted from the UML model. This happens because C\&A applications modeled 


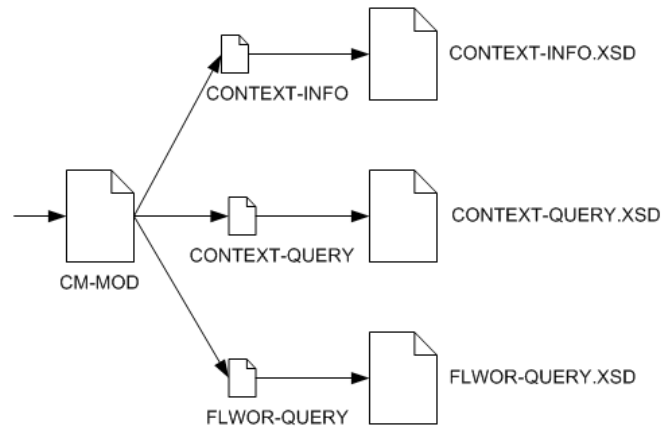

Figure 5: XML Schema Generation.

using the Contextractor methodology present a particular composition relationship that joins external and internal classes. In the case of our example, the name of this relationship is has, and, as can be seen in Figure 1, it is the point of junction between internal and external classes of the model.

The classes joined by the point of junction are Class and Course. It does make sense, as the event we aim at capturing is a class of a given course.

Therefore, the starting point for the generation of the CONTEXTINFO schema is the definition of the element what. The stylesheet responsible for defining this element searches the CM-MOD document for a composition relationship that has an internal class as a starting point and an external class as an ending point. After finding this relationship - there is only one - the element what is defined as a complex type containing the external class, with its attributes, nesting the internal class, which keeps only its identifier attribute.

For the remaining elements, the processing is guided by the attribute $c t x$, which holds a context dimension and is present in all attribute elements. Therefore, for each context dimension, an element is a complex type composed by elements named after the class names that present a ctx attribute with the same name of the processed dimension. These nested elements are themselves declared as complex types composed by an attribute having the same name as the attribute element that matched the context dimension name. The following piece of code illustrates the definition of the elements what and who.

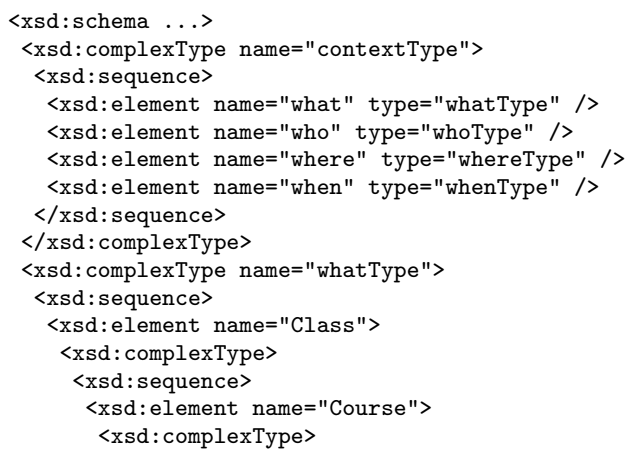

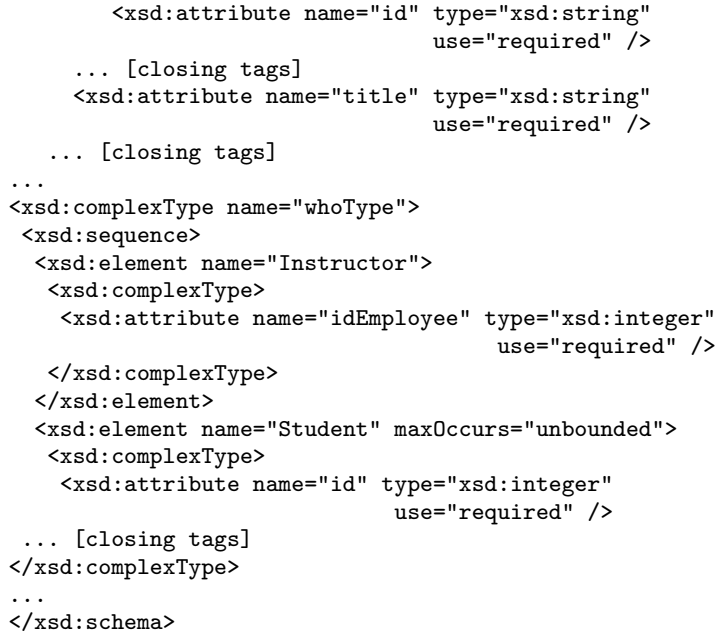

The relationship multiplicities present in the modified conceptual model are relevant to the modeling of the context schemas. The relationship multiplicities in our example indicate that many students may watch a class, while only one instructor can teach a class.

Those facts must be considered in the definition of the XML schemas, as relationship multiplicity determines the values of the minOccurs and maxOccurs attributes of the schema's elements. We adjust the number of occurrences of each element by analyzing the relationship multiplicities of the internal class of the point of junction and the other internal classes. When those relationships present multiplicities many to many or one to many, the elements associated with the classes that participates in those relationships must be modelled with maxOccurs="unbounded", meaning that several occurrences of this element may appear in the instance documents. This is exactly what happens in the definition of the element who, presented in the code fragment above. The multiplicity of the relationship enrolls caused the definition of the element Student with an unbounded number of occurrences.

The CONTEXT-INFO schema, produced as a result of this transformation process is used to validate instance documents as the example that follows. Note that the elements and attributes that define each context dimension were all obtained from the UML application model.

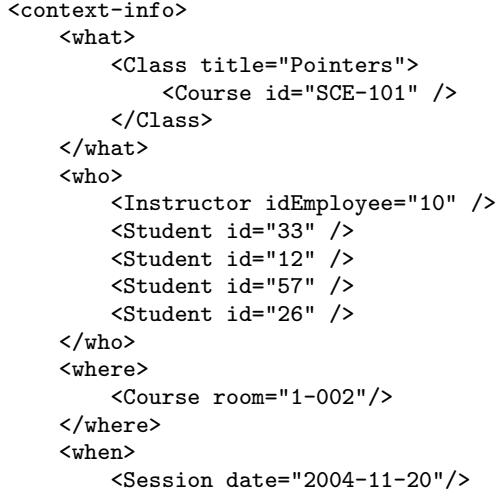




\author{
<Course term="20_2004" /> \\ $</$ when $>$ \\ $</$ context-info $>$
}

Figure 5 shows that, besides the CONTEXT-INFO, two other XML schemas are produced by the transformation process: CONTEXT-QUERY and FLWOR-QUERY. The first schema is used to define a query language called CQL - Contextual Query Language. The second is used by a program called CQL Processor, which is responsible by retrieving references to XML documents that obey the restrictions imposed in the CQL query expression.

The Context Query Language was designed with the goal of making it easy to retrieve documents simply by selecting any combination of context dimensions. To make this language as flexible as possible, we allow the user to declare a dimension using any attribute from the associated class. The only restriction is that the attribute used may not be of a different dimension than the one that is being specified. In other words, it means that, for our current example, it is possible to use the $i d$ or the name of an instructor, when using the who dimension in the query expression.

Another important difference between the CONTEXT-INFO and the CONTEXT-QUERY schemas refers to the number of occurrences of each element. Taking as an example the Instructor element, because the UML model specifies that a given course has exactly one instructor, this element appears with minOccurs and maxOccurs values of 1 in the CONTEXT-INFO schema. However, it must be possible to search for classes thought for different instructors. So, the element Instructor must be declared with a maxOccurs value of unbounded in the CONTEXT-QUERY schema. This implies that the query expression fragment below will retrieve the classes taught by Maria OR Dilvan.

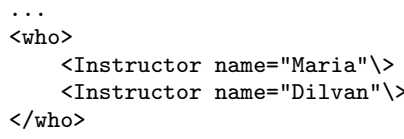

However, there may be cases in which the connector $A N D$ must be used. For example, the user may want to retrieve the sessions in which the students Renan, Valter and Carlos were present. Therefore, the CONTEXT-QUERY schema must provide a way for the user to specify the connector to be used in a query that contains two or more elements of the same class.

Our approach to deal with this issue uses the multiplicity of relationships that involve the internal class of the point of junction and the other internal classes. If a class presents multiplicity many in such a relationship, the elements in the CONTEXT-INFO schema are modelled with the extra attribute connector that may contain $A N D$ or $O R$.

Therefore, the semantics of the connection of identical elements follows the rule of thumb: if the element does not specify a connector attribute, this connector should be $O R$. Otherwise, the specified connector should be used.
The last difference between the CONTEXT-INFO and the CONTEXT-QUERY schemas refers to the occurrences of the context-dimension elements, which are all declared optional. This allows queries with any combination of dimensions.

The last schema produced by the Contextractor methodology is called FLWOR-QUERY. This schema is named after the name of query expressions in the XQuery Language [7]. Query expressions valid according to this schema are ready to be transformed to XQuery expressions and submitted to the document repository.

The FLWOR-QUERY and the CONTEXT-QUERY are both used by a query processor that gets as input CQL queries and returns references to documents that obeys the specified restrictions.

The FLWOR-QUERY schema is almost identical to the CONTEXT-INFO schema. There are only two differences: the first is the value of the minOccurs attribute of the dimension elements, which is set to 0 in the FLWOR-QUERY. The second is related to the connector attribute, which is used in the FLWOR-QUERY schema as it is used in the CONTEXT-QUERY schema.

\section{INFRASTRUCTURE SUPPORT BASED ON DOCUMENT PROCESSING}

The aim of our work is to rise the level of abstraction in the development of $\mathrm{C} \& \mathrm{~A}$ applications. For this sake, we provide a component based infrastructure to support the development of these applications. Leveraging off the communication abstraction provided by InCA [15], we built a component-based infrastructure that both allows the rapid prototyping of C\&A applications and provides a set of services for the management of the data produced by those applications.

InCA, Infrastructure for Capture and Access, was a first step towards providing a common model for the development of capture and access applications. Its model is based on the separation of key design concerns related to the capture and access activity and the provision of a software architecture that expresses those concerns as software interfaces and modules. In the core of this architecture, there is a communication mechanism that allows software modules to communicate transparently to the application developer.

Building on top of InCA, we developed xInCA - extended InCA - a set of reusable software components comprising the most common functionalities demanded by C\&A applications. xInCA provides components for capturing interactions with whiteboards, web browsing and chat. Special components are also provided for capturing audio and video from live experiences. xInCA makes it possible to build a C\&A application simply through the arrangement of the desired components $[6,5,8]$.

After addressing the problem of recurrence of functionalities, our next step towards rising the level of abstraction in the construction of C\&A applications was targeted to the management of the data produced during capture sessions. In this field, our aim was to automate the generation of 
presentation documents, making it possible to present the contents of a captured session on the Web without human intervention.

Our approach to achieve this goal involves the definition of a set of XML schemas: one for each xInCA component, one for the application's context information, called CONTEXTINFO schema, and one for grouping all the other schemas, called SESSION schema. Those schemas are used as input for a data binding mechanism, producing Java classes responsible for encapsulating captured content. The CONTEXTINFO schema used in the data binding process is the one produced by the Contextractor methodology.

The classes produced by the binding mechanism became part of the infrastructure, being used for exchanging data between the many modules that provide the functionality required by C\&A applications. One of these modules, called Transducer, uses the communication abstractions provided to receive in real time the objects produced by the application's capture components. The received objects are integrated to a session object, which class was generated by the compilation of the session schema by the data binding mechanism. The contents send to the Transducer by the C\&A application include the context object, which class was also produced by the data binding process. By the end of the session, the Transducer executes an operation called marshaling in the session object, producing an XML document that represents the captured content [4].

The Transducer is part of a service called StRES - Storing, Retrieving and Extending Service - which is responsible for the management of a document repository where the XML documents that represent captured sessions are stored. A main feature of StRES is that it provides presentation facilities to C\&A applications. Using a Cocoon based application, StRES is able to browse document collections in the document repository, building HTML pages on the fly with the contents of each collection. This makes possible to browse the document collections for desired sessions. As StRES organizes the repository using a document specified by the application's developer, the nesting of collections present associated semantics.

For the case of our example, the collections are organized by terms, courses and, finally, captured classes. So, the Web application initially displays the terms that present captured classes. By selecting a term, the user visualizes the courses of that term. Again, by selecting a course, a new page is generated with the captured classes. At this time, each class presents different links, each of them corresponding to different visualization formats.

Therefore, StRES makes it possible to visualize a captured content on the Web right after the end of a session, without the need of human intervention. However, this facility presents the drawback of not providing flexibility: all that is offered is a browsing mechanism to navigate within the document repository.

The Contextractor represents our effort to address this problem, as it allows the definition of a contextual query language based on the application's data model.
Before the implementation of the Contextractor, the context schema used by StRES was fixed. So, besides being possible to define the entity person, it was not possible to associate roles with it, such as instructor and student. Therefore, searches based on context dimensions were not very effective.

Using the Contextractor, however, it is possible to define schemas with associated semantics inherited from the application's conceptual model, allowing the definition of rich contextual query languages.

Exploiting the capabilities provided by the Contextractor, we extended our Web application providing a way for the user to specify a contextual query expression, which is used as input for the CQL processor. As a result, the user visualizes a page containing the sessions that obey the restrictions imposed by the query. Figure 6 presents different links for each session, allowing their visualization in different presentation formats.

\section{CQL PROCESSOR}

The functionality behind the Web application shown in Figure 6 is provided by a processor that takes as input a contextual query expression and returns a list of references for the documents that obey the restrictions imposed in the query.

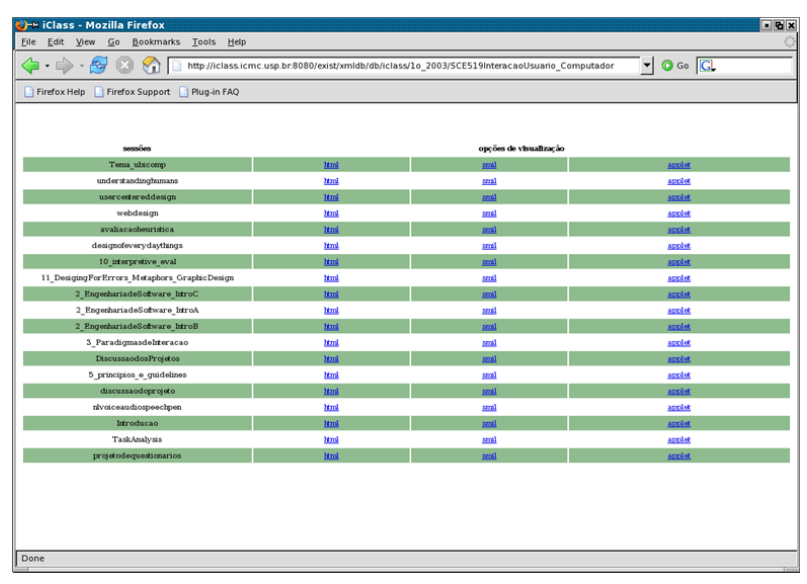

Figure 6: Visualization options

As we have mentioned in Section 4.3, the processing of a CQL query is based on both the CONTEXT-QUERY and the FLWOR-QUERY schemas. The former is used to validate the CQL query - only valid queries can be processed. The later is used to prepare the query so as it can be transformed in a FLWOR query and submitted to the XML document repository.

The first action of the CQL processor when a query is submitted is trying to validate the query expression (actually, an XML document) against the CONTEXT-QUERY schema. If the validation fails, an exception is raised and the processing is terminated. Otherwise, it is a valid CQL query and the processing may continue.

In the following phase, the expression must be validated using the FLWOR-QUERY schema. When the validation process fails, it is necessary to transform the query until the 
validation problem is solved. Lets take the following query expression as an example.

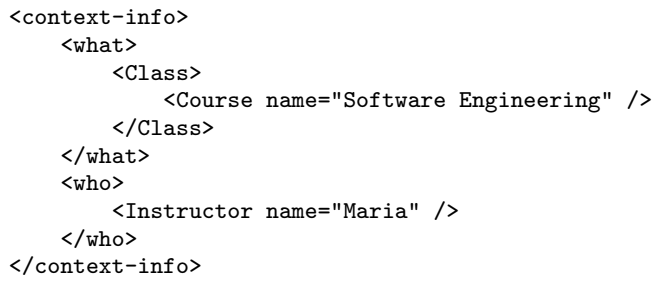

This is a valid query according to the CONTEXT-QUERY schema, but not valid according to FLWOR-QUERY. The validation problem is related to the attribute name: instance documents present the attribute $i d$. The CQL processor deals with this by exploiting the XML processing facilities provided by the Java language.

During the parsing of a document associated with an XML schema, validation errors are reported by a callback mechanism that identifies the element and/or attribute causing the error - showing what was expected and what was found. Using this information, the CQL processor is able to retrieve the information that is missing from the external repository (a legacy database for instance).

In the case of our example, the parser will identify a problem with the attribute name of the element Course. The generated error message will indicate that it was expected an attribute called $i d$. Using the information retrieved from the error message, the CQL processor is able to build the SQL query shown bellow.

SELECT id from Course WHERE name="Software Engineering";

After obtaining the value of the $i d$ attribute, the CQL processor updates the query with the correct attribute name and its associated value. The same process is restarted and finishes only when the document is valid according to the FLWOR-QUERY schema.

In the last step, the CQL processor transforms the context query in FLWOR query expression, which is then submitted to the document repository. We use the eXist native XML database as our document repository, as it provides support for the XQuery Language.

\section{RELATED WORK}

Various approaches have been used to model context information. Gu et al. [10] divide those approaches into applicationoriented, model-oriented and ontology-oriented. A more detailed study, conducted by Strang and Linnhoff-Popien [14], classified the modeling approaches using six different categories: key-value models, markup scheme models, graphical models, object-oriented models, logic-based models and ontology-based models.

Regardless of the modeling approach, the resulting models are usually part of frameworks, toolkits or infrastructures which aim to facilitate the development of context-aware applications. Integrated with development environments, the models define the representation - structure and content - of entities such as people and places, allowing common operations to store, retrieve and interchange context information.

Most of these models address the needs of ubiquitous computing applications for smart environments, where sensors and actuators are commonly used to monitor the situational state of people and objects. Processing information produced by sensors and triggering actions using actuators are main concerns of those applications and, therefore, those concerns must be accommodated by supporting models.

For C\&A applications context information may be processed during either the capture phase, the access phase, or both. In instrumented environments used for capturing live experiences, such as classrooms and meeting rooms, the acquisition of context information is easy: authentication procedures used by applications that manage capture devices make it easy to identify the people involved in the experience. Issues related to user location are also simplified, as mobility is limited to the instrumented rooms. So, the use of sensors and actuators does not substantially enrich C\&A applications.

Consequently, the modeling of context information for C\&A applications aims primarily to support the retrieval of captured information based on well-known concepts, such as the identification of people involved, the type of captured experiences, their location and time.

Besides the particularities presented by C\&A applications, it is possible to find similarities between the Contextractor and other modeling approaches found in the literature. Strang and Linnhoff-Popien [14] would classify the Contextractor as using agraphical model because it relies on UML class diagrams.

Among projects that provide graphical facilities for context modeling, the work conducted by Henricksen et al. [13] is particularly relevant. Built upon a previous graphical model called ORM - Object-Role Modeling [11] — the work of Henricksen and her colleagues provides a language for modeling context information called CML - Context Modeling Language. CML allows the formal specification of context requirements for context-aware applications. By means of a transformation mechanism it is possible to map a CML model to a context management system supported by a relational database. We notice similarities between Henricksen's work and the Contextractor, especially when considering the mapping approach used to produce the underlying storage model. Besides the mapping similarities, both approaches extend previous modeling tools to provide facilities for dealing with context data.

\section{CONCLUSIONS AND FUTURE WORK}

Context modeling has become an important concern in ubiquitous computing research, as it can be seen from the growing number of research projects and publications on the topic [14]. However, the existing and ongoing efforts do not address an important class of ubiquitous computing appli- 
cations, namely capture and access applications.

Those applications present different requirements from traditional ubiquitous applications, because they have two distinct phases where context is used. Some existing models present adequate support for the capture phase. However, those models are not adequate to support retrieval of captured content during the access phase.

To address this gap, we developed the Contextractor, a context modeling system that provides graphical facilities for the specification of context information. The modeling task using Contextractor provides a context query language that supports the interaction with the content produced by the modeled application. Therefore, each modeled application presents its own query language, which presents associated semantics, as their entities are build from the application's conceptual model specified in the UML class diagram.

To process the resulting query language we provide a program that processes as input a query expression and generates references to documents that obey the restrictions imposed by the query. This is a novel approach to modeling and processing context information. The results are positive: the interface for submitting queries produces the response page in a reasonable time for a Web application.

One clear limitation of the current approach is that the query expression must be written as an XML document. We plan to address this problem providing a graphical interface in which the user may interact with buttons to select elements and attributes and specify values for them, so that the query document is created implicitly.

\section{REFERENCES}

[1] G. Abowd and E. Mynatt. Charting past, present, and future research in ubiquitous computing. $A C M$ Transactions on Computer-Human Interaction, $7(1): 29-58,2000$.

[2] A. R. Andrade, E. Munson, and M. G. C. Pimentel. A document based approach to the generation of web applications. In Proceedings of the ACM Symposium on Document Engineering 2004, pages 45-47, 2004.

[3] A. R. Andrade, E. Munson, and M. G. C. Pimentel. Engineering web applications with XML and XSLT. In WebMedia \& $L A W e b 2004$ Joint Conference, pages 86-93. IEEE CS Press, 2004.

[4] L. A. Baldochi, A. R. Andrade, R. G. Cattelan, and M. G. C. Pimentel. Architecture and components for capture and access applications. In WebMedia \&6 $L A W e b 2004$ Joint Conference, pages 150-157. IEEE CS Press, 2004.

[5] L. A. Baldochi, R. G. Cattelan, and M. G. Pimentel. Building a middleware infrastructure for capture and access applications. In Proceedings of the $X X X$ SEMISH - Integrated Seminar on Software and Hardware, pages 299-313, 2003.

[6] L. A. Baldochi, R. G. Cattelan, M. G. C. Pimentel, and K. N. Truong. Automatic generation of capture and access applications. In Proceedings of the 8th
Brazilian Symposium on Multimedia and Hypermedia Systems, pages 100-115, 2002.

[7] S. Boag, D. Chamberlin, M. F. Fernández, D. Florescu, J. Robie, and J. Siméon. Xquery 1.0: An XML query language. W3C Working Draft http://www.w3.org/TR/xquery/, October 2004.

[8] R. G. Cattelan, L. A. Baldochi, and M. G. C. Pimentel. Processing and storage middleware support for capture and access applications. In Companion Proceedings of the 2003 ACM/IFIP/USENIX International Middleware Conference, page 315, 2003

[9] R. G. Cattelan, L. A. Baldochi, M. G. C. Pimentel, and K. N. Truong. Experiences on building capture and access applications. In Proceedings of the 9th Brazilian Symposium on Multimedia and Hypermedia Systems, pages 112-127, 2003.

[10] T. Gu, X. H. Wang, H. K. Pung, and D. Q. Zhang. An ontology-based context model in intelligent environments. In Communication Networks and Distributed Systems Modeling and Simulation Conference, 2004.

[11] T. A. Halpin. Conceptual Schema and Relational Database Design. Prentice Hall Australia, 2nd edition, 1995.

[12] S. Helal. Programming pervasive spaces. IEEE Pervasive, 4(1):84-87, 200.

[13] K. Henricksen and J. Indulska. A software engineering framework for context-aware pervasive computing. In Proceedings of the Second IEEE International Conference on Pervasive Computing and Communications (PerCom 2004), pages 77-86. IEEE Computer Society, 2004.

[14] T. Strang and C. Linnhoff-Popien. A context modeling survey. In Workshop on Advanced Context Modelling, Reasoning and Management as part of UbiComp 2004 - The Sixth International Conference on Ubiquitous Computing, 2004.

[15] K. Truong and G. Abowd. Inca: A software infrastructure to facilitate the construction and evolution of ubiquitous capture \& access applications. In Proceedings of the Second International Conference on Pervasive Computing, pages 140-157, 2004.

[16] K. N. Truong, G. D. Abowd, and J. A. Brotherton. Personalizing the capture of public experiences. In UIST '99: Proceedings of the 12th annual ACM symposium on User interface software and technology, pages 121-130. ACM Press, 1999.

[17] R. Want, B. Schilit, N. Adams, R. Gold, K. Petersen, J. Ellis, D. Goldberg, and M. Weiser. The parctab ubiquitous computing environment. Technical Report CSL-95-1, Xerox PARC, 1995.

[18] M. Weiser. The computer for the 21st century. Scientific American, 265(3):94-104, 1991. 